\title{
Inspecter sans inspecteur
}

Le cas du Danemark

\section{Peter Weng}

\section{OpenEdition}

\section{Journals}

Édition électronique

URL : http://journals.openedition.org/ries/3561

DOI : $10.4000 /$ ries.3561

ISSN : 2261-4265

Éditeur

Centre international d'études pédagogiques

\section{Édition imprimée}

Date de publication : 1 décembre 1995

Pagination : 141-142

ISSN : 1254-4590

Référence électronique

Peter Weng, «Inspecter sans inspecteur », Revue internationale d'éducation de Sèvres [En ligne] 08 | 1995, mis en ligne le 13 février 2014, consulté le 21 avril 2019. URL : http:// journals.openedition.org/ries/3561; DOI : 10.4000/ries.3561

Ce document a été généré automatiquement le 21 avril 2019

(c) Tous droits réservés 


\title{
Inspecter sans inspecteur
}

\author{
Le cas du Danemark
}

\section{Peter Weng}

1 La notion « d'inspection » doit être traitée en relation avec l'élaboration des programmes et l'enseignement dans les écoles municipales (Folkeskolen'1) et les lycées (Gymnasium²).

2 Au Danemark, c'est le Parlement qui définit les buts de l'école municipale et le ministre de l'éducation qui établit les objectifs dans chaque discipline. Mais ces buts et ces objectifs ne sont indiqués que dans les grandes lignes. C'est donc chaque école qui, dans ce cadre, élabore le plan détaillé. Ensuite, le conseil municipal doit approuver ce plan et veiller à ce que l'école respecte les grandes lignes fixées pour toutes les écoles du pays.

3 Il est à noter que, même si le ministère publie des guides sur l'enseignement et les programmes, ils n'ont qu'une valeur indicative et non contraignante; l'enseignant peut utiliser la méthode qu'il considère la meilleure et choisir un contenu différent de celui suggéré dans le guide, à condition que cette méthode satisfasse aux exigences du ministère. En conséquence, on trouve de nombreuses façons d'enseigner la même discipline dans les écoles municipales. Cette liberté dont jouit l'enseignant dans le choix de ses méthodes d'enseignement constitue un droit culturel, rendant très rare le contrôle direct d'une classe par un inspecteur.

4 La stratégie de contrôle, mise en place par le Parlement et le ministère est axée sur les objectifs et le cadre, tandis que les élus locaux en liaison avec les écoles ont la charge de la réalisation de ces objectifs dans le détail.

5 Ces dernières années, l'octroi des subventions aux écoles dépend en partie du nombre d'élèves dans l'école et ces subventions sont attribuées en bloc, ce qui permet aux écoles de disposer d'une liberté financière, puisque le contrôle financier par le ministère des détails du plan, du contenu des disciplines, du nombre des classes, etc., a été réduit.

6 Le remplacement du contrôle de l'État par les structures en place avec davantage de liberté financière découle du principe que le contrôle le plus efficace ne vient pas des autorités centrales ou des élus locaux, mais du personnel lui-même dans les écoles, quand 
il s'agit de l'amélioration de l'enseignement et du profil scolaire des élèves, l'école gardant ainsi une réputation de qualité.

7 En 1990, on a institué un conseil d'école dans les Folkeskolen. Le rôle du conseil d'école est d'établir les principes visant à développer les activités de l'école selon les plans approuvés par le conseil municipal. Le conseil d'école dont la majorité des membres sont des parents représentant les usagers de l'école, décide de l'organisation de l'enseignement, du nombre de cours dans chaque discipline, etc. En outre, le conseil approuve le budget et l'équipement pédagogique. Le conseil d'école peut donc avoir une influence et une responsabilité considérables en ce qui concerne l'enseignement au sein de la classe. Mais, dans la pratique, la question est de savoir combien de conseils utilisent cette possibilité.

Le chef d'établissement est chargé de l'administration de l'école et a l'entière responsabilité éducative. Cela signifie que le chef d'établissement doit aller dans les classes observer le travail de l'enseignant, si celui-ci est un débutant ou s'il a des raisons de penser que l'enseignement ne s'effectue pas tel qu'il a été planifié.

En général, le contexte culturel et traditionnel est tel qu'il n'y a pas de contrôle au quotidien du travail de l'enseignant.

10 C'est seulement en cas de plainte des parents ou d'autres personnes que le chef d'établissement ou une autre autorité intervient dans le travail de l'enseignant. Les enseignants ne veulent pas être contrôlés et ils considèrent une telle démarche comme une atteinte à leur honneur et à leur intégrité.

11 En liaison avec la nouvelle loi pour la Folkeskole entrée en vigueur en 1994, le Parlement a décidé d'encourager et de promouvoir les innovations. Un stage spécial de formation de six semaines a été organisé à l'intention de cent quatre-vingts enseignants sélectionnés qui ont été mis à la disposition des écoles dans tout le pays pour aider à la mise en place de cette nouvelle loi.

12 Au niveau des Gymnasium, des représentants du ministère se rendent sur place au moins une fois par an. Le visiteur peut être un expert d'une discipline ou un inspecteur pédagogique. Normalement, ces personnes assistent à un certain nombre de cours afin d'avoir une impression d'ensemble de l'enseignement et de faire des propositions en vue d'en améliorer la qualité. Mais, parfois, la visite est motivée par l'intervention du chef d'établissement qui, ayant l'impression que le travail d'un enseignant ne répond pas aux exigences, fait appel au consultant pour venir assister aux cours de ce professeur.

13 En conclusion, on peut dire que la fonction "d'inspection" dans les écoles danoises correspond à la coopération entre le chef d'établissement, les enseignants, les parents et les élèves.

14 Si les uns et les autres utilisent leurs droits démocratiques aux différents niveaux du système éducatif, il y a de grandes chances pour qu'un tel enseignement réponde aux exigences de la législation ministérielle. 


\section{NOTES}

1. Folkestolen : école accueillant tous les élèves au cours de la scolarité obligatoire (de 5 à 16 ans), selon un tronc commun d'enseignement.

2. Gymnasium : établissement d'enseignement secondaire post-obligatoire.

\section{RÉSUMÉS}

Le système éducatif danois laisse une grande autonomie aux établissements dans l'organisation de leur enseignement de base (Folkeskolen). La régulation des établissements repose sur une coopération étroite entre le chef d'établissement, les parents et les élèves.

\section{INDEX}

Index géographique : Danemark

Mots-clés : autonomie, chef d'établissement, inspection, relation parents-école, inspecteur

\section{AUTEUR}

\section{PETER WENG}

Chercheur, Danish National Institute for Educational Research, Copenhague, Danemark.

Sylvie Bronner 